\title{
In Vitro Characterization of the OptiChamber Diamond Valved Holding Chamber
}

\author{
Ross H.M. Hatley, PhD, Dirk von Hollen, BS, ${ }^{2}$ Dennis Sandell, PhLic, ${ }^{3}$ and Lois Slator, MSc ${ }^{1}$
}

\begin{abstract}
Background: Use of a valved holding chamber (VHC) in conjunction with a pressurized metered dose inhaler (pMDI) can reduce issues relating to poor actuation-inhalation coordination and potentially improve the lung deposition of aerosol, compared with use of a pMDI alone. However, the performance of a VHC is influenced by different device-related factors, including the size and shape of the VHC and the material it is manufactured from (conventional versus antistatic). This study aimed to provide an in vitro characterization of an antistatic VHC, the OptiChamber Diamond VHC, comparing the aerodynamic particle size distribution of aerosol delivered via this VHC with results from a second antistatic VHC and a conventional VHC.

Methods: The pMDI drug formulations (albuterol, suspension; beclomethasone dipropionate, solution) were connected to a Next Generation Impactor, either directly (pMDI alone tests) or via a VHC (VHC tests). The pMDIs were actuated ( $\times 10$ per product pair) and tested at extraction flow rates of $15 \mathrm{~L} / \mathrm{min}$ and $30 \mathrm{~L} / \mathrm{min}$, without any time delay between actuation and inhalation. Dose delivery using the two pMDI drug formulations was compared, and is presented with reference to key aerodynamic particle size parameters.

Results: Compared with tests on pMDIs alone, use of a VHC increased the dose of aerosol within the respirable range, particularly at a $15 \mathrm{~L} / \mathrm{min}$ flow rate. Between-VHC comparisons indicated that the two antistatic VHCs were equivalent. Delivery of albuterol appeared to be influenced by the VHC used, but beclomethasone dipropionate seemed unaffected.

Conclusions: The two antistatic VHCs were equivalent for both pMDI brands. Aerosol delivered from the antistatic VHCs at $15 \mathrm{~L} / \mathrm{min}$ had a higher proportion of fine particles compared with the conventional VHC.
\end{abstract}

Key words: valved holding chamber, flow rate, in vitro equivalence, pMDI, aerodynamic particle size distribution

\section{Introduction}

$\mathbf{V}$ ALVED HOLDING CHAMBERS (VHCs) are accessory devices for pressurized metered dose inhalers (pMDIs) that were developed in response to patient-related difficulties with the optimal use of pMDIs. ${ }^{(1)}$ In vitro work suggests that use of a VHC may reduce the impact of poor actuationinhalation coordination, even if the actuation-inhalation is completely mistimed. ${ }^{(2)}$ Clinical studies have shown that use of a contemporary hydrofluoroalkane (HFA) pMDI with a VHC can increase lung deposition and attenuate oropharyngeal and gastrointestinal deposition, compared with delivery of the same formulation via a dry powder inhaler, ${ }^{(3,4)}$ whereas in vitro work indicates that use of a pMDI with VHC can reduce oropharyngeal deposition relative to the pMDI alone. $^{(5)}$ Collectively, such benefits have the potential to improve the targeting of aerosol to the lungs while reducing local side effects.

The principle function of a VHC is to provide space for the development of the aerosol plume, deceleration of droplets, evaporation of propellant, and gravimetric deposition of larger, nonrespirable particles. Together, these factors may increase the efficiency of aerosol delivery and reduce the likelihood of local side effects. It has long been recognized that valves confer to a VHC the ability to "hold" aerosol, and thus reduce the need for perfect

\footnotetext{
${ }^{1}$ Respironics Respiratory Drug Delivery (UK) Ltd., a business of Philips Electronics UK Limited, Chichester, West Sussex, UK.

${ }^{2}$ Respironics, Inc., a Philips Healthcare company, Murrysville, PA, USA.

${ }^{3}$ S5 Consulting, Ekvägen 8, S-275 62 Blentarp, Sweden.
} 
actuation-inhalation coordination. ${ }^{(6)}$ Previous work using gamma scintigraphy indicated that use of a VHC, as an accessory to a contemporary pMDI, can result in favorable lung deposition, regardless of whether the patient performs a breathing maneuver (slow inhalation followed by breath hold) or inhales in tidal breaths. ${ }^{(3)}$

Despite the apparently simple holding function, numerous VHC specifications have been shown to influence delivered dose and fine particle characteristics, and must be considered in the design and manufacturing process. Factors known to influence the output from a VHC include valve design, ${ }^{(7,8)}$ the presence of electrostatic charge on the surface of the $\mathrm{VHC},{ }^{(9)}$ and, although it remains controversial, the size and shape of the VHC. ${ }^{(1)}$

The OptiChamber Diamond VHC [Diamond; Respironics Respiratory Drug Delivery (UK) Ltd., a business of Philips Electronics UK Limited, Chichester, West Sussex, UK) is an antistatic VHC made from acrylonitrile butadiene styrene, with an antistatic polymer additive that facilitates the dissipation of localized electrostatic charge over its surface. VHCs manufactured from such antistatic materials are less prone to the accumulation of static charge and may provide a more consistent dose of drug. ${ }^{(9)}$ The Diamond VHC has a volume of $140 \mathrm{~mL}$ and a length of $14.2 \mathrm{~cm}$, and can be disassembled for hand washing. The Diamond VHC has both an inspiratory valve and an expiratory valve. The inspiratory valve is a low-resistance, low dead-space, duck-billed valve, designed to minimize any impediment to air flow, which might otherwise lead to high levels of local aerosol deposition. The exhalation valve is clearly visible, which allows caregivers to view the deflection of the valve; this provides feedback on the patient's breath count and breath holding. Compliance-related feedback is provided via a warning whistle, which signals an excessively high inhalation flow rate. In addition to a stepped mouthpiece, designed to facilitate the transfer of pediatric patients to use of the mouthpiece, the Diamond VHC can be fitted with the LiteTouch facemask [Respironics Respiratory Drug Delivery (UK) Ltd.], suitable for young patients who inhale tidally from the VHC. The LiteTouch facemask incorporates soft-seal technology, which is designed to provide a high-quality seal, at minimal application pressure, with low leakage.

The Diamond VHC is marketed under $510(\mathrm{k})$ clearance in the United States, having been determined to be "substantially equivalent" to the AeroChamber Plus Z-Stat VHC (AC Z-Stat; Monaghan Medical Corp., Plattsburgh, NY) by the US Food and Drug Association. The Diamond VHC is also CE marked for use in Europe.

In this article, in vitro aerosol characteristics of the antistatic Diamond VHC, a second antistatic VHC, and an older, conventional VHC are compared. The experiments were carried out using two different drug formulations commonly prescribed to respiratory patients, and assessed key delivery parameters that are typically used to define the characteristics of aerosols and aerosol delivery devices. Results are presented with reference to these key parameters. The delivery of a suspension of albuterol and a solution of beclomethasone dipropionate was investigated, in order to ensure that the evaluation compared both solution and suspension drug formulations that are commonly used in the treatment of asthma. In addition, the antistatic performance of the Diamond VHC was confirmed by characterizing the fine particle distribution of aerosol delivered via both an unwashed and a washed Diamond VHC. To ensure the applicability of results to patients who inhale from a VHC in tidal breaths, investigations were carried out at $15 \mathrm{~L} / \mathrm{min}$ and $30 \mathrm{~L} /$ min extraction flow rates. No delay between actuation and the start of inhalation flow was used in the present study.

\section{Materials and Methods}

\section{Test products}

Two HFA pMDI drug formulations, a suspension of albuterol (albuterol; ProAir $90 \mu \mathrm{g}$ HFA; Teva Respiratory LLC, Horsham, PA) and a solution of beclomethasone dipropionate (beclomethasone; QVAR $100 \mu \mathrm{g}$ HFA; Teva Specialty Pharmaceuticals), were evaluated using a Next Generation Impactor (NGI; MSP Corporation, St. Paul, MN).

ProAir is supplied as a pressurized canister, with a red plastic actuator and white dust cap. Each canister weighs $8.5 \mathrm{~g}$ and provides 200 actuations (NDC 59310-579-20). On actuation, $120 \mu \mathrm{g}$ of albuterol is delivered from the canister valve, and $108 \mu \mathrm{g}$ is delivered from the actuator mouthpiece; this is equivalent to $90 \mu \mathrm{g}$ of albuterol base.

QVAR is supplied in a pressurized canister, with a dark mauve plastic actuator and a gray dust cap. Each canister weighs $7.3 \mathrm{~g}$ and contains 100 actuations (NDC 59310-17780). On actuation, $100 \mu \mathrm{g}$ of beclomethasone dipropionate is delivered from the canister valve, and $80 \mu \mathrm{g}$ is delivered from the actuator mouthpiece. Neither of the pMDIs tested contained a dose counter.

Drug delivery characteristics of albuterol and beclomethasone delivered via the pMDI alone, the Diamond VHC, the AeroChamber Plus VHC (AC Plus; Monaghan Medical Corp.), and the AC Z-Stat VHC were compared.

\section{Pretest conditioning}

All pMDIs, VHCs, test equipment, and fluids were stabilized to ambient conditions for $2 \mathrm{hr}$ prior to use. The pMDI actuator was cleaned before use and before each test run during the study (washed in warm soapy water, rinsed with deionized water, and air-dried). The pMDI was primed by firing a number of shots to waste, as stipulated in the instructions for use, supplied with each pMDI brand.

Prior to use and during the study, the US Pharmacopeia (USP) induction port was washed in warm soapy water, rinsed with deionized water, rinsed with methanol, and left to air-dry. All the VHCs were washed, rinsed, and dried before each test, with the exception of the unwashed Diamond VHCs, which were kept in their original packaging until just before testing.

A mixed anionic/nonionic detergent (15-30\% anionic surfactants, 5-15\% nonionic surfactants) was used to create the warm soapy water in which all above components were washed (Fairy Liquid, Proctor \& Gamble UK, Weybridge, Surrey, UK).

\section{NGI preparation}

The NGI was operated as per British Standard EN 13544$1: 2007^{(10)}$ with either a $15 \mathrm{~L} / \mathrm{min}$ or a $30 \mathrm{~L} / \mathrm{min}$ air flow through the system. These flow rates were chosen to reflect the range of typical tidal inhalation flow rates observed in patients. $^{(10)}$ 
The effect of NGI plate coating on particle bounce was considered; however, given that this analysis concerned differences in VHC performance, any effect of particle bounce was not thought to be critical to the primary objectives of the study. Air flow was generated by a Copley HCP5 vacuum pump (Copley Scientific Ltd., Nottingham, UK). The vacuum pump was connected to a backup filter placed on the exhaust port of the NGI. A TSI mass flowmeter (TSI Inc., Shoreview, MN) was sealed in line between the pMDI actuator and the USP induction port, with the arrow on the bottom of the meter pointing in the direction of the extraction flow. Once the correct extraction flow had been set, the mouthpiece of the pMDI actuator was connected to the USP induction port and sealed with Parafilm M (Bemis Company, Inc., Oshkosh, WI).

\section{Test plan}

The two pMDI drug formulations were assessed at two different flow rates, giving a total of four different pMDI-flow rate combinations. The tests were conducted according to a set schedule, designed to minimize any potential systematic error caused by dose trend throughout the life of the pMDI (Table 1). Each of the four tests used six pMDI canisters, with the exception of albuterol at $15 \mathrm{~L} / \mathrm{min}$ (three canisters). For each test, six runs were performed per pMDI, with 10 actuations per run. Given that this study was designed to investigate between-VHC variability, 10 actuations per run were chosen, in order to minimize variability conferred by the pMDI. This number of actuations also ensured that the dose collected in each NGI stage reached the lower limit of quantification.

The test schedule for albuterol at $30 \mathrm{~L} / \mathrm{min}$ involved six runs, to allow for the use of both a washed and an unwashed Diamond VHC. This constrained the test schedule design; the washed Diamond VHC tests were conducted with the same devices as the unwashed, which necessitated that washed Diamond VHC tests followed tests using unwashed Diamond VHCs. All references to the "Diamond VHC" refer to the device in the washed state, unless otherwise specified.

\section{Tests on pMDI alone}

Before use, each pMDI was primed according to the instructions given in the relevant prescribing information (ProAir, $3 \times$ actuations, shaking well before each actuation; QVAR, $2 \times$ actuations). The pMDI mouthpiece was connected to the USP induction port, as described above (NGI preparation); the pMDI canister was removed from the actuator, shaken as required, returned to the actuator, and immediately actuated (held down for $1-2 \mathrm{sec}$ ). This was repeated a further nine times with a 20 -sec delay between repeats. The extraction flow through the NGI was run for a further $10 \mathrm{sec}$ after the final actuation/20-sec delay period. The USP induction port, NGI stages, and backup filter were eluted with a suitable diluent (albuterol, 10\% acetonitrile solution; beclomethasone, $60 \%$ acetonitrile solution). A predetermined quantity of diluent was used for each component (USP induction port, $10 \mathrm{~mL}$; backup filter, $25 \mathrm{~mL}$; NGI, $10 \mathrm{~mL}$ per stage). The amount of drug in each eluate was quantified using high-performance liquid chromatography (HPLC; see below, HPLC analysis). The USP induction port and pMDI actuator were cleaned in preparation for the subsequent test.

\section{Tests on VHCs}

For the VHC tests, the NGI was assembled with a clean set of stages, and a leak test was performed. The USP induction port was fitted to the NGI inlet, and a backup filter was connected to the exhaust port. The NGI was set up as previously detailed (NGI preparation). The mouthpiece of the VHC was connected to the USP induction port using an elastomeric-lipped ISO connector (Intersurgical Ltd., Wokingham, UK). This connection was sealed with Parafilm M, so as to prevent leakage. The pMDI actuator was fitted to the VHC; the pMDI canister was then removed from the plastic actuator, prepared according to the instructions given in the relevant prescribing information, returned to the actuator, and immediately actuated (held down for $1-2 \mathrm{sec}$ ). This was repeated a further nine times with a 20 -sec delay between repeats. The extraction flow through the NGI was run for a further $10 \mathrm{sec}$ after the last actuation/20-sec delay period.

The USP induction port, NGI stages, and backup filter were eluted with a suitable diluent (albuterol, 10\% acetonitrile solution; beclomethasone, $60 \%$ acetonitrile solution). Given that this study focused on ex-actuator dose, drug deposited on the pMDI actuator was not eluted. A predetermined quantity of diluent was used for each component (USP induction port, $10 \mathrm{~mL}$; backup filter, $25 \mathrm{~mL}$; NGI, $10 \mathrm{~mL}$ per stage; VHC, $30 \mathrm{~mL}$ ). The amount of drug in each eluate was quantified using HPLC (see below, HPLC analysis). The mouthpiece of the VHC was sealed with Parafilm M, and a quantity of diluent was dispensed into the VHC. The back end cap of the VHC was then sealed with Parafilm M and the VHC was shaken, so that all internal surfaces were fully washed. The back end cap was unsealed, and the diluent was transferred to a collection vessel. Eluate obtained was quantified using HPLC. The USP induction port and pMDI actuator were cleaned in preparation for the subsequent test.

\section{HPLC analysis}

Following each NGI run, all test components were covered and kept dry, until processed. Individual components were processed separately: each component was washed with diluent, and a sample was placed in the HPLC vial, prior to washing the next component.

The HPLC equipment (Agilent 1100 series; Agilent Technologies, Waldbronn, Germany) was fitted with the appropriate column [albuterol, Genesis Phenyl, $4 \mu \mathrm{m}, 150 \times$ $4.5 \mathrm{~mm}, 22^{\circ} \mathrm{C}, 1 \mathrm{~mL} / \mathrm{min}$; beclomethasone, Phenomenex Luna $\left.\mathrm{C} 18(2), \quad 3 \mathrm{~mm}, \quad 150 \times 4.5 \mathrm{~mm}, 25^{\circ} \mathrm{C}, 1 \mathrm{~mL} / \mathrm{min}\right]$. Bracketing standards were made up and run, and the accuracy of the standards was checked. System suitability was then confirmed using five replicate injections of the bracketing standard to be used throughout the run. In order for the results of the run to be valid, the relative standard deviation of the bracketing standards had to be below $2 \%$ throughout the run, and the tailing factor (USP method) had to be no greater than predefined values (albuterol, 2.0; beclomethasone, 1.5).

\section{Statistical analysis}

The primary objectives of the statistical analysis, for both pMDI drug formulations, were to compare:

- Results for drug delivery via the VHCs to drug delivery via the pMDIs alone 
Table 1. Test Schedule

\begin{tabular}{|c|c|c|c|c|c|c|c|}
\hline \multirow[b]{2}{*}{ Run } & \multicolumn{6}{|c|}{ VHC type } & \multirow[b]{2}{*}{ Actuations } \\
\hline & Test 1 & Test 2 & Test 3 & Test 4 & Test 5 & Test 6 & \\
\hline 0 & Prime & Prime & Prime & Prime & Prime & Prime & 3 \\
\hline 1 & pMDI 1 & pMDI 2 & pMDI 3 & pMDI 4 & pMDI 5 & pMDI 6 & $1-10$ (10 shots/filter) \\
\hline 2 & $\begin{array}{l}\text { Diamond } \\
\text { (unwashed) }\end{array}$ & AC Plus & AC Z-Stat & $\begin{array}{l}\text { Diamond } \\
\text { (unwashed) }\end{array}$ & AC Plus & $\begin{array}{l}\text { Diamond } \\
\text { (unwashed) }\end{array}$ & 12-21 (10 shots/filter) \\
\hline 3 & AC Z-Stat & $\begin{array}{l}\text { Diamond } \\
\text { (unwashed) }\end{array}$ & AC Plus & Diamond & $\begin{array}{l}\text { Diamond } \\
\text { (unwashed) }\end{array}$ & AC Z-Stat & 23-32 (10 shots/filter) \\
\hline 4 & Diamond & AC Z-Stat & $\begin{array}{l}\text { Diamond } \\
\text { (unwashed) }\end{array}$ & AC Plus & AC Z-Stat & Diamond & 34-43 (10 shots/filter) \\
\hline 5 & AC Plus & Diamond & Diamond & AC Z-Stat & Diamond & AC Plus & 45-54 (10 shots/filter) \\
\hline 6 & pMDI & pMDI & pMDI & pMDI & pMDI & pMDI & 56-65 (10 shots/filter) \\
\hline
\end{tabular}

- Aerodynamic particle size distribution of aerosol delivered via the different VHC brands

- The effect of the two different flow rates

- Any differences between drug delivery via a washed Diamond VHC and an unwashed Diamond VHC

- The delivery of albuterol and beclomethasone

Comparisons were made for the following endpoints:

- Delivered dose $(\mathrm{DD})=($ Throat + Stage $1+$ Stage $2+\ldots+$ Stage $7+$ micro-orifice collector + backup filter $)$

- Fine particle dose $\leq 5 \mu \mathrm{m}$ (FPD)

- Fine particle dose $\leq 1 \mu \mathrm{m}$ (FPD1)

- Mass median aerodynamic diameter (MMAD)

- Dose recovered from VHC

- Fine particle fraction $\leq 5 \mu \mathrm{m}(\mathrm{FPF})=100 \times \mathrm{FPD} /(\mathrm{DD}+$ dose recovered from $\mathrm{VHC}$ )

Note that delivered dose, FPD, and FPD1 were normalized to the label claim for delivered dose (ProAir albuterol, $90 \mu \mathrm{g}$; QVAR beclomethasone, $80 \mu \mathrm{g}$ ) in order to achieve a common scale and allow comparison between pMDI drug formulations. FPD and FPD1 were calculated from stage data, using linear interpolation on the original scale between the relevant adjacent stages. MMAD was determined using Copley Inhaler Testing Data Analysis Software (CITDAS V3.10, Copley Scientific Ltd., Nottingham, UK).

The delivery method (pMDI alone, AC Z-Stat VHC, AC Plus VHC, Diamond VHC) was treated as a fixed factor, with a random intercept and a random slope included in the model to adjust for possible within-canister trends. A separate model was used for each combination of key variable and pMDI product. The mean values (least square-means) were estimated, together with the relevant differences. A log transformation of the key variables was used to calculate the relative differences and confidence intervals. In vitro equivalence between "Test" and "Reference" devices can be claimed when the $90 \%$ confidence interval for the mean ratio of the endpoint under study is within the acceptance interval of $85-118 \%$ (i.e., a maximal difference of $15 \%)^{(11)}$

\section{Results}

\section{Delivered dose}

The delivered dose for the pMDI alone and each VHC, at $15 \mathrm{~L} / \mathrm{min}$ and $30 \mathrm{~L} / \mathrm{min}$ flow rate, is shown in Figure 1. The

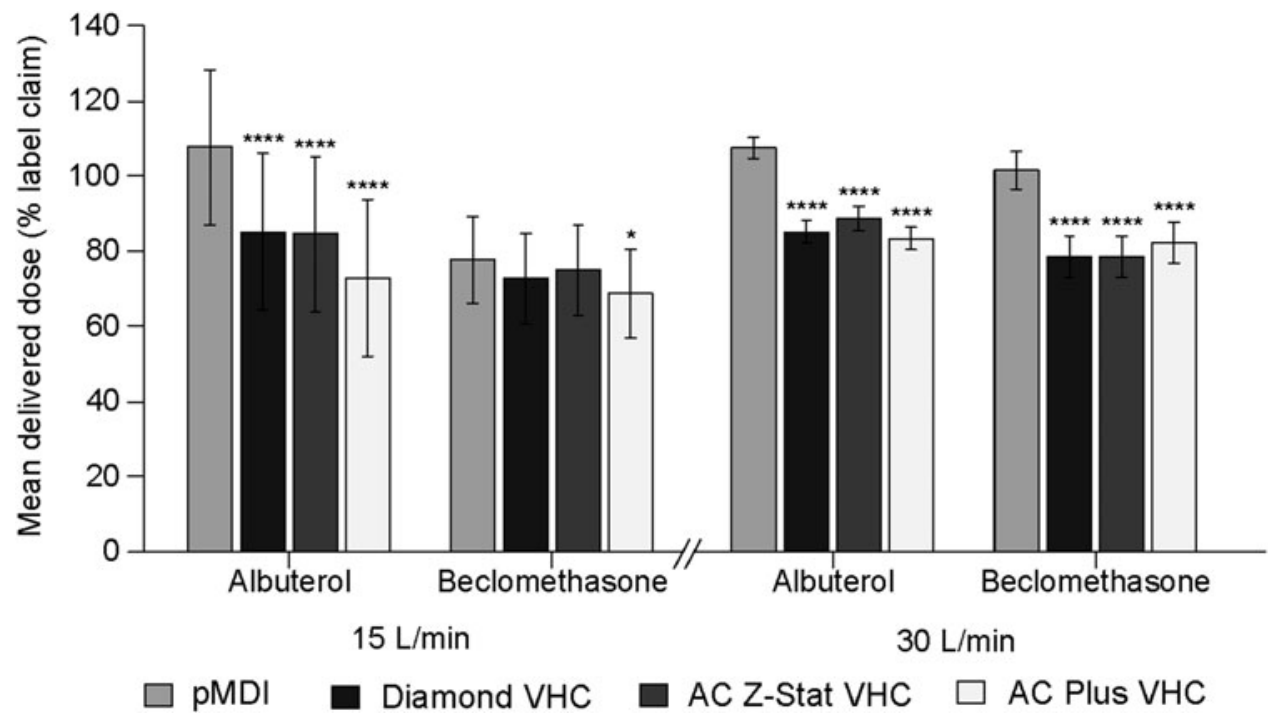

FIG. 1. Estimated mean delivered dose as percentage of label claim. Error bars represent $90 \%$ confidence intervals about the mean. All comparisons are with pMDI alone; $* p<0.05, * * * * p<0.0001$. 
Table 2. Estimated Mean Relative Difference in Delivered Dose (\% Label Claim), and 90\% Confidence Limits, BY pMDI PRODUCT, FlOW RATE, AND VHC COMPARISON

\begin{tabular}{|c|c|c|c|c|c|c|}
\hline $\begin{array}{l}\text { pMDI } \\
\text { product }\end{array}$ & $\begin{array}{c}\text { Flow } \\
\text { rate } \\
\text { (L/min) }\end{array}$ & Device comparison & $\begin{array}{c}\text { Estimated } \\
\text { difference }(\% \text { LC) }\end{array}$ & $\begin{array}{c}\text { Lower } 90 \% \\
\text { confidence } \\
\text { limit }(\%)\end{array}$ & $\begin{array}{c}\text { Upper } 90 \% \\
\text { confidence } \\
\text { limit (\%) }\end{array}$ & $\mathrm{p}$ value \\
\hline \multirow[t]{6}{*}{ Albuterol } & \multirow[t]{3}{*}{15} & Diamond vs. pMDI alone & 0.79 & 0.75 & 0.83 & $<0.0001$ \\
\hline & & AC Z-Stat vs. pMDI alone & 0.78 & 0.74 & 0.83 & $<0.0001$ \\
\hline & & AC Plus vs. pMDI alone & 0.68 & 0.64 & 0.71 & $<0.0001$ \\
\hline & \multirow[t]{3}{*}{30} & Diamond vs. pMDI alone & 0.79 & 0.77 & 0.82 & $<0.0001$ \\
\hline & & AC Z-Stat vs. pMDI alone & 0.82 & 0.80 & 0.85 & $<0.0001$ \\
\hline & & AC Plus vs. pMDI alone & 0.77 & 0.75 & 0.80 & $<0.0001$ \\
\hline \multirow[t]{6}{*}{ Beclomethasone } & \multirow[t]{3}{*}{15} & Diamond vs. pMDI alone & 0.92 & 0.83 & 1.02 & 0.1847 \\
\hline & & AC Z-Stat vs. pMDI alone & 0.94 & 0.85 & 1.05 & 0.3455 \\
\hline & & AC Plus vs. pMDI alone & 0.87 & 0.79 & 0.97 & 0.0329 \\
\hline & \multirow[t]{3}{*}{30} & Diamond vs. pMDI alone & 0.77 & 0.72 & 0.81 & $<0.0001$ \\
\hline & & AC Z-Stat vs. pMDI alone & 0.77 & 0.73 & 0.82 & $<0.0001$ \\
\hline & & AC Plus vs. pMDI alone & 0.81 & 0.76 & 0.86 & $<0.0001$ \\
\hline
\end{tabular}

$\%$ LC, \% label claim.

mean delivered dose of albuterol achieved using the pMDI alone was significantly greater than that achieved with the use of any of the three VHCs (Table 2). This pattern was observed at both $15 \mathrm{~L} / \mathrm{min}$ and $30 \mathrm{~L} / \mathrm{min}$ flow rates. The delivered dose of beclomethasone followed a similar pattern with a flow rate of $30 \mathrm{~L} / \mathrm{min}$; a significantly lower delivered dose was achieved in the presence of a VHC compared with the pMDI alone. This is a predictable effect of using a pMDI with a VHC.

The delivery of beclomethasone with a flow rate of $15 \mathrm{~L} /$ min was the exception to this pattern; the delivered dose of beclomethasone achieved with either of the antistatic VHCs did not differ significantly from that achieved with the
FIG. 2. FPD (A) and FPD1 (B) as percentage of label claim. Error bars represent 90\% confidence intervals about the mean. All comparisons are with pMDI alone; $* p<0.05, \quad * * * p<0.001$, $* * * * p<0.0001$.
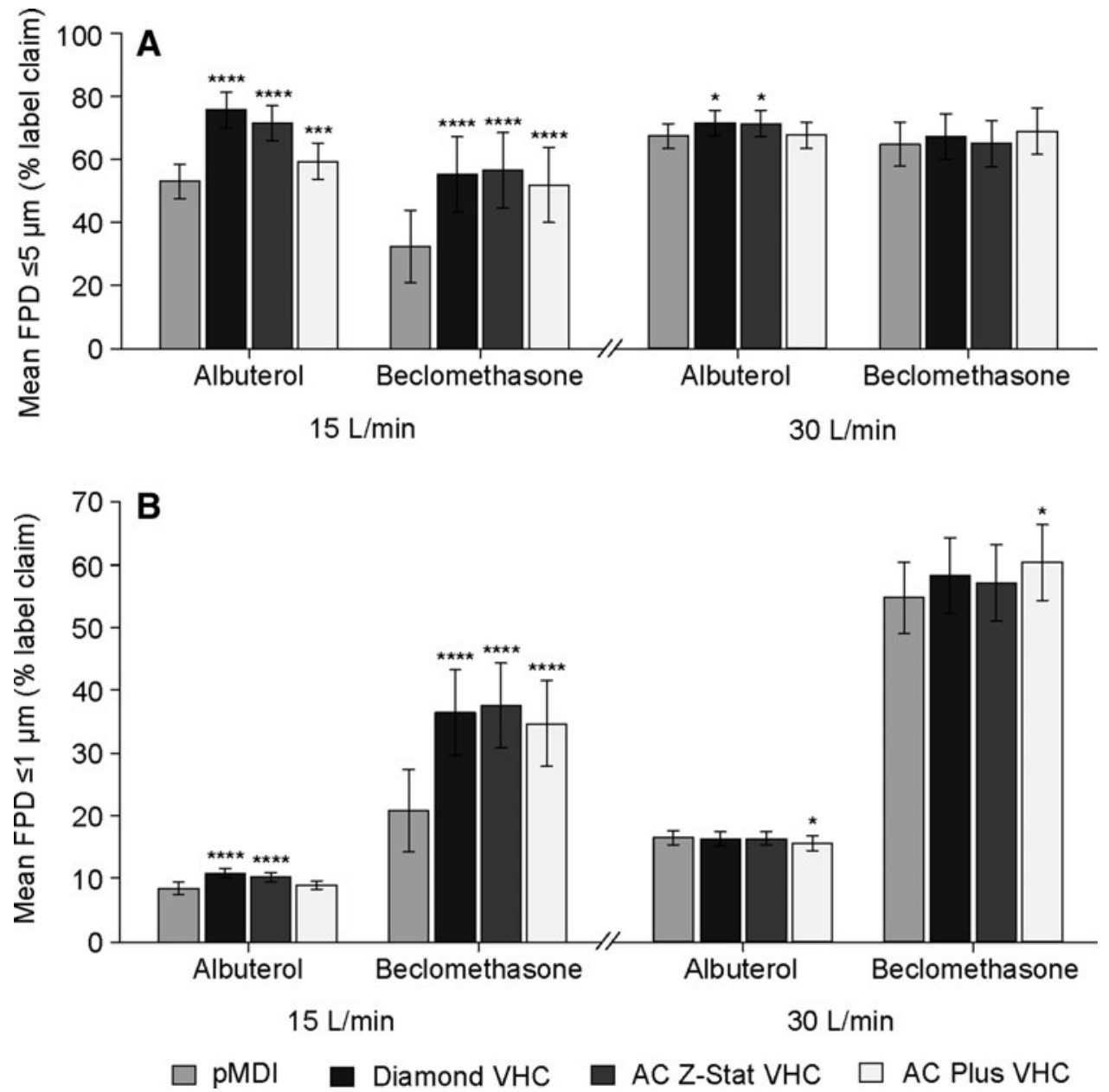
Table 3. Estimated Mean Relative Difference in FPD5 (\% Label Claim), and 90\% Confidence Limits, BY pMDI PRODUCT, FLOW RATE, AND VHC COMPARISON

\begin{tabular}{|c|c|c|c|c|c|c|}
\hline $\begin{array}{l}\text { pMDI } \\
\text { product }\end{array}$ & $\begin{array}{l}\text { Flow } \\
\text { rate } \\
(\text { L/min })\end{array}$ & Device comparison & $\begin{array}{c}\text { Estimated } \\
\text { difference } \\
(\% \text { LC) }\end{array}$ & $\begin{array}{l}\text { Lower } 90 \% \\
\text { confidence } \\
\text { limit }(\%)\end{array}$ & $\begin{array}{l}\text { Upper } 90 \% \\
\text { confidence } \\
\text { limit }(\%)\end{array}$ & $\mathrm{p}$ value \\
\hline \multirow[t]{2}{*}{ Albuterol } & 15 & $\begin{array}{l}\text { Diamond vs. pMDI alone } \\
\text { AC Z-Stat vs. pMDI alone } \\
\text { AC Plus vs. pMDI alone }\end{array}$ & $\begin{array}{l}1.43 \\
1.35 \\
1.12\end{array}$ & $\begin{array}{l}1.39 \\
1.31 \\
1.09\end{array}$ & $\begin{array}{l}1.48 \\
1.40 \\
1.16\end{array}$ & $\begin{aligned} &<<0.0001 \\
&<0.0001 \\
& 0.0002\end{aligned}$ \\
\hline & 30 & $\begin{array}{l}\text { Diamond vs. pMDI alone } \\
\text { AC Z-Stat vs. pMDI alone } \\
\text { AC Plus vs. pMDI alone }\end{array}$ & $\begin{array}{l}1.06 \\
1.05 \\
1.00\end{array}$ & $\begin{array}{l}1.02 \\
1.02 \\
0.96\end{array}$ & $\begin{array}{l}1.10 \\
1.09 \\
1.04\end{array}$ & $\begin{array}{l}0.0125 \\
0.0229 \\
0.9424\end{array}$ \\
\hline \multirow[t]{2}{*}{ Beclomethasone } & 15 & $\begin{array}{l}\text { Diamond vs. pMDI alone } \\
\text { AC Z-Stat vs. pMDI alone } \\
\text { AC Plus vs. pMDI alone }\end{array}$ & $\begin{array}{l}1.68 \\
1.70 \\
1.55\end{array}$ & $\begin{array}{l}1.48 \\
1.50 \\
1.37\end{array}$ & $\begin{array}{l}1.90 \\
1.93 \\
1.75\end{array}$ & $\begin{array}{l}<0.0001 \\
<0.0001 \\
<0.0001\end{array}$ \\
\hline & 30 & $\begin{array}{l}\text { Diamond vs. pMDI alone } \\
\text { AC Z-Stat vs. pMDI alone } \\
\text { AC Plus vs. pMDI alone }\end{array}$ & $\begin{array}{l}1.03 \\
1.00 \\
1.06\end{array}$ & $\begin{array}{l}0.96 \\
0.93 \\
0.99\end{array}$ & $\begin{array}{l}1.10 \\
1.07 \\
1.14\end{array}$ & $\begin{array}{l}0.5046 \\
0.9211 \\
0.1332\end{array}$ \\
\hline
\end{tabular}

$\%$ LC, \% label claim.

pMDI alone, whereas use of the AC Plus VHC was associated with a significantly lower delivered dose. Overall, there was a greater variability in delivered dose with a flow rate of $15 \mathrm{~L} / \mathrm{min}$ compared with $30 \mathrm{~L} / \mathrm{min}$.

Fine particle dose $\leq 5 \mu \mathrm{m}$

Drug recovered in particles with an aerodynamic diameter $\leq 5 \mu \mathrm{m}$ (FPD), at $15 \mathrm{~L} / \mathrm{min}$ and $30 \mathrm{~L} / \mathrm{min}$, is shown in Figure $2 \mathrm{~A}$. With an extraction flow rate of $15 \mathrm{~L} / \mathrm{min}$, use of each of the VHCs resulted in a significantly greater FPD of albuterol compared with the pMDI alone (Table 3). A significant increase in the FPD of albuterol was also observed at $30 \mathrm{~L} / \mathrm{min}$, although this was restricted to the antistatic VHCs. The FPD of beclomethasone delivered via each brand of $\mathrm{VHC}$ at $15 \mathrm{~L} / \mathrm{min}$ was significantly greater than that achieved via the pMDI alone. In contrast to albuterol, at a $30 \mathrm{~L} / \mathrm{min}$ flow rate, there was no difference in the FPD of beclomethasone achieved using a VHC compared with that achieved with the pMDI alone. Notably, between-VHC differences for both albuterol and beclomethasone were more pronounced at the lower flow rate.

\section{Fine particle dose $\leq 1 \mu \mathrm{m}$}

The mean FPD1 of albuterol and beclomethasone delivered at $15 \mathrm{~L} / \mathrm{min}$ and $30 \mathrm{~L} / \mathrm{min}$ flow rates, relative to the label claim, are shown in Fig. 2B. Mean FPD1 of albuterol delivered at $15 \mathrm{~L} / \mathrm{min}$ via the Diamond VHC and the AC Z-Stat VHC was significantly greater than that delivered via the pMDI alone (Table 4). There was no significant difference between the FPD1 of albuterol delivered via the AC Plus VHC at $15 \mathrm{~L} / \mathrm{min}$ and that delivered with the pMDI alone. There was no significant difference in the FPD1 of albuterol delivered via the Diamond VHC and AC Z-Stat VHC at 30 L/ min compared with pMDl alone. When the AC Plus VHC was used to deliver albuterol at $30 \mathrm{~L} / \mathrm{min}$, the FPD1 was marginally lower; this difference was statistically significant.

Table 4. Estimated Mean Relative Difference in FPD1 (\% Label Claim), and 90\% Confidence Limits, BY pMDI PRODUCT, Flow RATE, AND VHC COMPARISON

\begin{tabular}{lclccrr}
\hline $\begin{array}{l}\text { pMDI } \\
\text { product }\end{array}$ & $\begin{array}{c}\text { Flow } \\
\text { rate } \\
(\text { L/min) }\end{array}$ & \multicolumn{1}{c}{ Device comparison } & $\begin{array}{c}\text { Estimated } \\
\text { difference } \\
(\% \text { LC })\end{array}$ & $\begin{array}{c}\text { Lower 90\% } \\
\text { confidence } \\
\text { limit }(\%)\end{array}$ & $\begin{array}{c}\text { Upper 90\% } \\
\text { confidence } \\
\text { limit (\%) }\end{array}$ & p value \\
\hline Albuterol & \multirow{2}{*}{15} & Diamond vs. pMDI alone & 1.28 & 1.20 & 1.35 & 0.0002 \\
& & AC Z-Stat vs. pMDI alone & 1.20 & 1.14 & 1.28 & 0.0008 \\
& & AC Plus vs. pMDI alone & 1.06 & 1.00 & 1.12 & 0.0976 \\
& \multirow{2}{*}{30} & Diamond vs. pMDI alone & 0.99 & 0.95 & 1.03 & 0.6107 \\
& & AC Z-Stat vs. pMDI alone & 0.99 & 0.95 & 1.03 & 0.7041 \\
Beclomethasone & & AC Plus vs. pMDI alone & 0.94 & 0.91 & 0.98 & 0.0167 \\
& \multirow{2}{*}{15} & Diamond vs. pMDI alone & 1.74 & 1.56 & 1.94 & $<0.0001$ \\
& & AC Z-Stat vs. pMDI alone & 1.78 & 1.60 & 1.99 & $<0.0001$ \\
& & AC Plus vs. pMDI alone & 1.62 & 1.45 & 1.81 & $<0.0001$ \\
& \multirow{2}{*}{30} & Diamond vs. pMDI alone & 1.05 & 0.99 & 1.12 & 0.1644 \\
& & AC Z-Stat vs. pMDI alone & 1.04 & 0.97 & 1.10 & 0.3379 \\
& & AC Plus vs. pMDI alone & 1.10 & 1.03 & 1.17 & 0.0168 \\
\hline
\end{tabular}

$\%$ LC, \% label claim. 
FIG. 3. MMAD by pMDI drug formulation, $\mathrm{VHC}$, and flow rate. Error bars represent $90 \%$ confidence intervals about the mean. All comparisons are with pMDI alone; ${ }^{*} p<0.05$, ** $p<0.01$, $* * * p<0.001$.

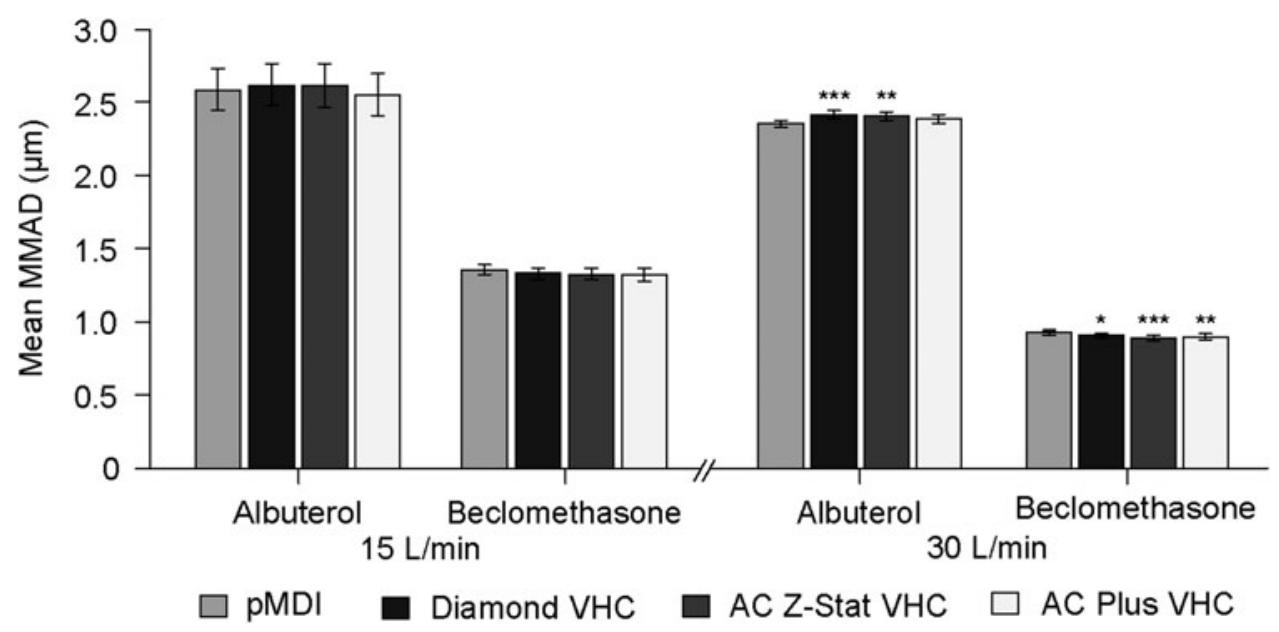

At a flow rate of $15 \mathrm{~L} / \mathrm{min}$, a substantially greater FPD1 of beclomethasone was delivered compared with the observed FPD1 for albuterol. The FPD1 of beclomethasone was significantly greater when delivered via any of the VHCs tested compared with that delivered via the pMDI alone. In contrast, at $30 \mathrm{~L} / \mathrm{min}$, a statistically significant increase in FPD1 was observed only with the AC Plus VHC compared with the pMDI alone. In general, the FPD1 of both albuterol and beclomethasone increased approximately twofold with a $30 \mathrm{~L} / \mathrm{min}$ flow compared with the $15 \mathrm{~L} / \mathrm{min}$ flow, regardless of the VHC used.

\section{Mass median aerodynamic diameter}

The MMAD for albuterol and beclomethasone, delivered at both $15 \mathrm{~L} / \mathrm{min}$ and $30 \mathrm{~L} / \mathrm{min}$, is shown in Figure 3. Overall variation in the MMAD was low, regardless of the presence of a VHC or the type of VHC used. MMAD of albuterol delivered via the VHCs at a $15 \mathrm{~L} / \mathrm{min}$ flow rate did not differ from that delivered via the pMDI alone (Table 5). At $30 \mathrm{~L} /$ min, there was a significant increase in MMAD when albuterol was delivered via the Diamond VHC and the AC ZStat VHC compared with the pMDI alone. A similar pattern was observed for beclomethasone; with a $15 \mathrm{~L} / \mathrm{min}$ flow rate, use of a VHC was not associated with a change in MMAD compared with the pMDI alone. When flow rate increased to $30 \mathrm{~L} / \mathrm{min}$, the MMAD of beclomethasone delivered via each of the VHCs was fractionally lower than that observed with the pMDI alone; these differences were statistically significant.

\section{Fine particle fraction $\leq 5 \mu \mathrm{m}$}

The fine particle fraction $\leq 5 \mu \mathrm{m}$ (FPF) for albuterol and beclomethasone, delivered at $15 \mathrm{~L} / \mathrm{min}$ and $30 \mathrm{~L} / \mathrm{min}$ flow rate, via each of the VHCs, is shown in Figure 4. At the lower flow rate, use of any of the three VHCs was associated with a significantly greater FPF of albuterol (Table 6). This difference was greater with the antistatic VHCs. At $30 \mathrm{~L} /$ min flow rate, only the antistatic Diamond VHC and AC ZStat VHC were associated with an increased FPF. For beclomethasone, the increase in $\mathrm{FPF}$ at $15 \mathrm{~L} / \mathrm{min}$ flow rate showed a similar pattern to that observed with albuterol. In contrast to albuterol, at $30 \mathrm{~L} / \mathrm{min}$, the AC Plus VHC was associated with a slight but significant increase in FPF relative to the pMDI alone.

Table 5. Estimated Mean Difference in MMAD ( $\mu \mathrm{M})$, and 90\% Confidence Limits, By pMDI Product, FLOW RATE, AND VHC COMPARISON

\begin{tabular}{|c|c|c|c|c|c|c|}
\hline $\begin{array}{l}\text { pMDI } \\
\text { product }\end{array}$ & $\begin{array}{c}\text { Flow } \\
\text { rate } \\
\text { (L/min) }\end{array}$ & Device comparison & $\begin{array}{c}\text { Estimated } \\
\text { difference } \\
(\% \text { LC) }\end{array}$ & $\begin{array}{c}\text { Lower } 90 \% \\
\text { confidence } \\
\text { limit }(\%)\end{array}$ & $\begin{array}{c}\text { Upper } 90 \% \\
\text { confidence } \\
\text { limit (\%) }\end{array}$ & $\mathrm{p}$ value \\
\hline \multirow[t]{6}{*}{ Albuterol } & \multirow[t]{3}{*}{15} & Diamond vs. pMDI alone & 1.01 & 0.99 & 1.04 & 0.2859 \\
\hline & & AC Z-Stat vs. pMDI alone & 1.01 & 0.99 & 1.04 & 0.3788 \\
\hline & & AC Plus vs. pMDI alone & 0.99 & 0.96 & 1.01 & 0.3473 \\
\hline & \multirow[t]{3}{*}{30} & Diamond vs. pMDI alone & 1.03 & 1.01 & 1.04 & 0.0009 \\
\hline & & AC Z-Stat vs. pMDI alone & 1.02 & 1.01 & 1.04 & 0.0064 \\
\hline & & AC Plus vs. pMDI alone & 1.01 & 1.00 & 1.03 & 0.0893 \\
\hline \multirow[t]{6}{*}{ Beclomethasone } & \multirow[t]{3}{*}{15} & Diamond vs. pMDI alone & 0.98 & 0.96 & 1.00 & 0.1636 \\
\hline & & AC Z-Stat vs. pMDI alone & 0.98 & 0.95 & 1.00 & 0.0897 \\
\hline & & AC Plus vs. pMDI alone & 0.97 & 0.95 & 1.00 & 0.0572 \\
\hline & \multirow[t]{3}{*}{30} & Diamond vs. pMDI alone & 0.98 & 0.96 & 0.99 & 0.0242 \\
\hline & & AC Z-Stat vs. pMDI alone & 0.96 & 0.94 & 0.98 & 0.0008 \\
\hline & & AC Plus vs. pMDI alone & 0.97 & 0.95 & 0.99 & 0.0085 \\
\hline
\end{tabular}

$\%$ LC, \% label claim. 


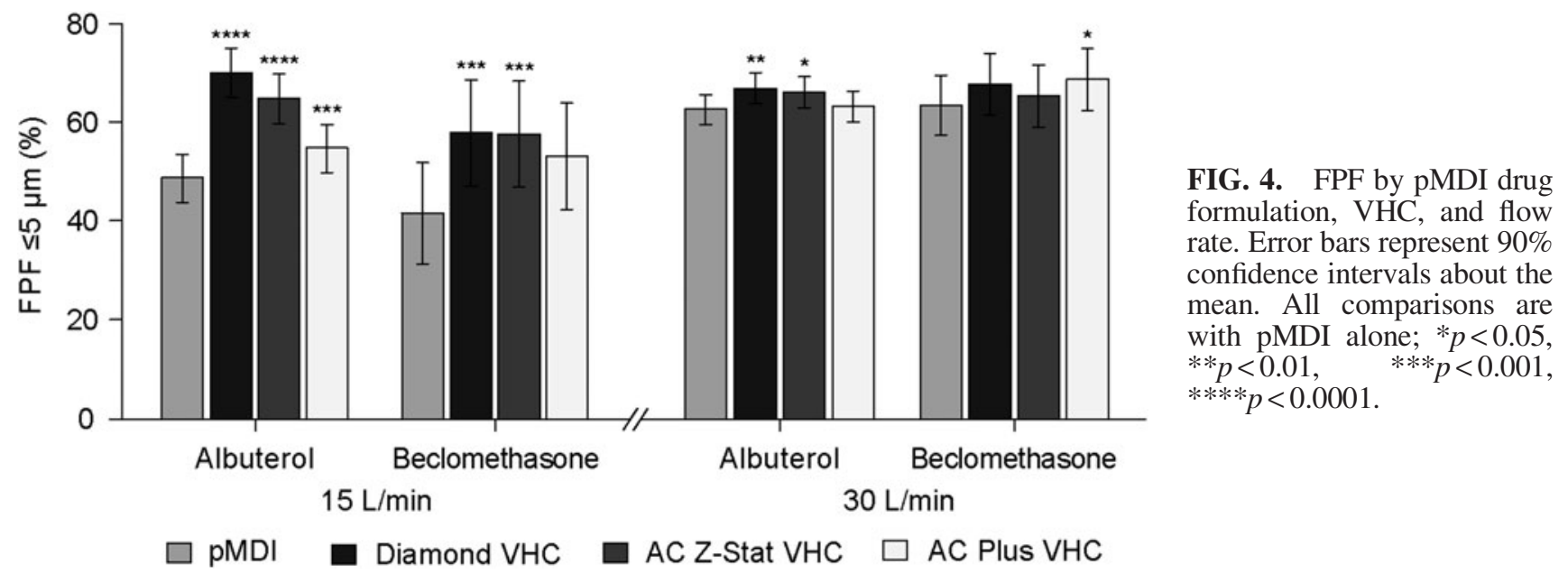

\section{Mean dose recovered from $\mathrm{VHC}$}

The dose of albuterol and beclomethasone recovered from the VHCs following delivery at $15 \mathrm{~L} / \mathrm{min}$ and $30 \mathrm{~L} / \mathrm{min}$ is shown in Figure 5. At $15 \mathrm{~L} / \mathrm{min}$, a significantly greater dose of albuterol was recovered from the AC Plus VHC compared with either the Diamond VHC or the AC Z-Stat VHC (Table 7). At $30 \mathrm{~L} / \mathrm{min}$, a significantly greater dose was recovered from the Diamond VHC compared with the AC ZStat VHC. Compared with the AC Plus VHC, a significantly lower dose was recovered from the AC Z-Stat VHC.

For beclomethasone, there was no significant difference between within-chamber loss of drug with the AC Z-Stat VHC compared with the Diamond VHC. A significantly greater quantity of drug was recovered from the AC Plus VHC compared with either of the antistatic VHCs. At $30 \mathrm{~L} /$ min, the percentage of the label claim of beclomethasone recovered from the VHCs did not differ significantly between VHC brands.

\section{VHC comparisons}

Between-VHC comparisons for the key parameters of albuterol and beclomethasone delivery, at $15 \mathrm{~L} / \mathrm{min}$ and
$30 \mathrm{~L} / \mathrm{min}$, are summarized in forest plots. The $90 \%$ confidence intervals for the ratio between Diamond VHC versus AC Z-Stat VHC (Fig. 6), Diamond VHC versus AC Plus VHC (Fig. 7), and AC Plus VHC versus AC Z-Stat VHC (Fig. 8) are shown; 90\% confidence intervals contained within the $85 \%$ and $118 \%$ bracket indicate in vitro equivalence. ${ }^{(11)}$ At $15 \mathrm{~L} / \mathrm{min}$, between-VHC comparisons indicate that a significantly greater delivered dose was achieved with the Diamond VHC compared with the AC Plus VHC $(p=0.0025)$. Use of the AC Plus VHC was also associated with a lower delivered dose compared with the AC Z-Stat VHC $(p=0.0036)$. There was no significant difference in the delivered dose achieved with the Diamond VHC compared with the AC Z-Stat VHC. There were no statistically significant between-VHC differences in the delivered dose of beclomethasone. Only the AC Plus VHC at $30 \mathrm{~L} / \mathrm{min}$ was associated with a slight, but statistically significant, reduction in the delivered dose of albuterol when compared with the AC Z-Stat VHC ( $p=0.0057)$. No other pairwise VHC comparisons differed significantly for the delivery of either albuterol or beclomethasone.

At $15 \mathrm{~L} / \mathrm{min}$, albuterol delivered via the Diamond VHC $(p<0.0001)$ and AC Z-Stat VHC $(p<0.0001)$ had a greater

Table 6. Estimated Mean Relative Difference in FPF (\%), and 90\% Confidence Limits, BY pMDI PRODUCT, Flow RATE, AND VHC COMPARISON

\begin{tabular}{|c|c|c|c|c|c|c|}
\hline $\begin{array}{l}\text { pMDI } \\
\text { product }\end{array}$ & $\begin{array}{l}\text { Flow } \\
\text { rate } \\
\text { (L/min) }\end{array}$ & Device comparison & $\begin{array}{l}\text { Estimated } \\
\text { difference } \\
(\% \text { LC) }\end{array}$ & $\begin{array}{l}\text { Lower } 90 \% \\
\text { confidence } \\
\text { limit }(\%)\end{array}$ & $\begin{array}{l}\text { Upper } 90 \% \\
\text { confidence } \\
\text { limit }(\%)\end{array}$ & $\mathrm{p}$ value \\
\hline \multirow[t]{2}{*}{ Albuterol } & 15 & $\begin{array}{l}\text { Diamond vs. pMDI alone } \\
\text { AC Z-Stat vs. pMDI alone } \\
\text { AC Plus vs. pMDI alone }\end{array}$ & $\begin{array}{l}1.44 \\
1.33 \\
1.13\end{array}$ & $\begin{array}{l}1.39 \\
1.29 \\
1.09\end{array}$ & $\begin{array}{l}1.49 \\
1.38 \\
1.16\end{array}$ & $\begin{array}{rl}< & 0.0001 \\
<0.0001 & 0.0002\end{array}$ \\
\hline & 30 & $\begin{array}{l}\text { Diamond vs. pMDI alone } \\
\text { AC Z-Stat vs. pMDI alone } \\
\text { AC Plus vs. pMDI alone }\end{array}$ & $\begin{array}{l}1.07 \\
1.06 \\
1.01\end{array}$ & $\begin{array}{l}1.04 \\
1.02 \\
0.98\end{array}$ & $\begin{array}{l}1.11 \\
1.09 \\
1.05\end{array}$ & $\begin{array}{l}0.0022 \\
0.0122 \\
0.5692\end{array}$ \\
\hline \multirow[t]{2}{*}{ Beclomethasone } & 15 & $\begin{array}{l}\text { Diamond vs. pMDI alone } \\
\text { AC Z-Stat vs. pMDI alone } \\
\text { AC Plus vs. pMDI alone }\end{array}$ & $\begin{array}{l}1.37 \\
1.37 \\
1.25\end{array}$ & $\begin{array}{l}1.21 \\
1.20 \\
1.10\end{array}$ & $\begin{array}{l}1.56 \\
1.55 \\
1.42\end{array}$ & $\begin{array}{l}0.0004 \\
0.0005 \\
0.0067\end{array}$ \\
\hline & 30 & $\begin{array}{l}\text { Diamond vs. pMDI alone } \\
\text { AC Z-Stat vs. pMDI alone } \\
\text { AC Plus vs. pMDI alone }\end{array}$ & $\begin{array}{l}1.06 \\
1.02 \\
1.08\end{array}$ & $\begin{array}{l}1.00 \\
0.97 \\
1.02\end{array}$ & $\begin{array}{l}1.12 \\
1.08 \\
1.14\end{array}$ & $\begin{array}{l}0.0959 \\
0.5261 \\
0.0269\end{array}$ \\
\hline
\end{tabular}

$\%$ LC, \% label claim. 
FIG. 5. Mean dose recovered from VHC (as percentage of label claim) by pMDI product, VHC, and flow rate. Error bars represent $90 \%$ confidence intervals about the mean. Arrows indicate between-VHC comparisons; $* p<0.05$, $* * * p<0.001$

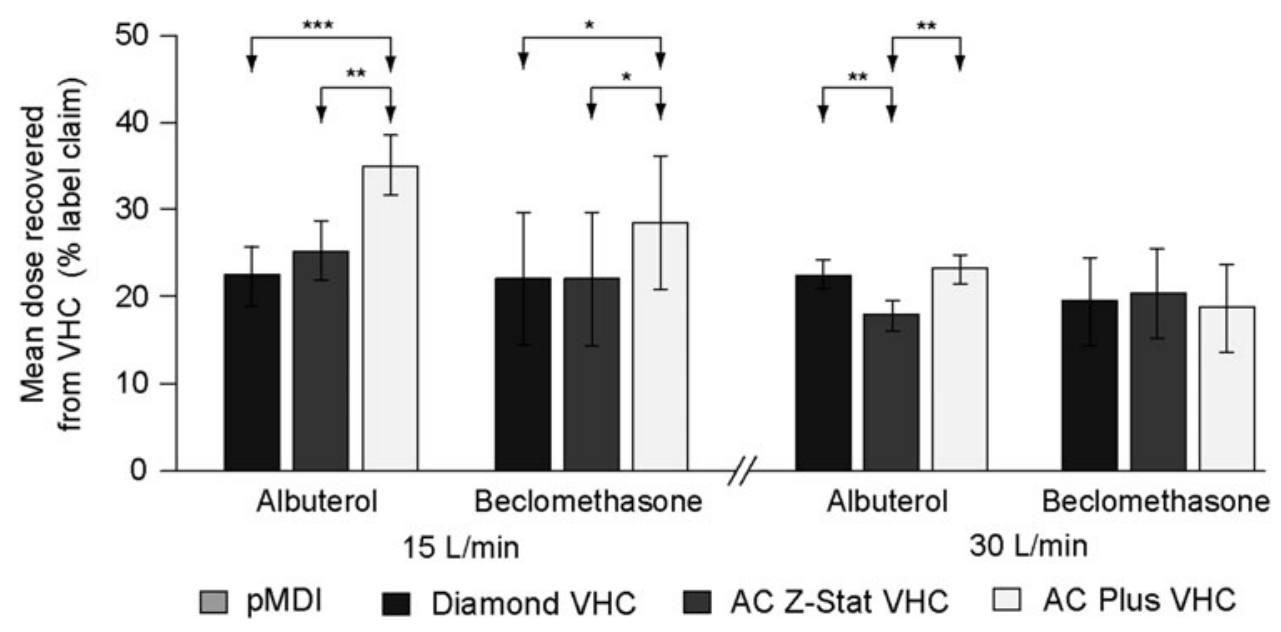

FPD compared with that delivered via the AC Plus VHC. There was a slight, but significant, increase in the FPD of albuterol delivered via the Diamond VHC compared with the AC Z-Stat VHC $(p=0.0223)$. At $30 \mathrm{~L} / \mathrm{min}$, there was a trend toward a lower FPD of albuterol when delivered via the AC Plus VHC compared with the AC Z-Stat VHC $(p=0.0578)$. For beclomethasone, no between-VHC differences in FPD were observed at either flow rate.

At $15 \mathrm{~L} / \mathrm{min}$, the FPD1 of albuterol delivered via the Diamond VHC did not differ from that delivered via the AC Z-Stat VHC. The FPD1 of drug delivered via the AC Plus VHC was significantly lower than that achieved with either the AC Z-Stat VHC $(p=0.0158)$ or the Diamond VHC $(p=0.0024)$. At $30 \mathrm{~L} / \mathrm{min}$, there were no significant between-VHC differences in albuterol FPD1. The FPD1 of beclomethasone did not differ depending on the VHC used, regardless of flow rate. There were no significant betweenVHC differences in the MMAD of either albuterol or beclomethasone, at either flow rate.

A greater FPF was achieved when albuterol was delivered via the Diamond VHC and the AC Z-Stat VHC compared with the AC Plus VHC (Diamond VHC, $p<0.0001$; AC ZStat VHC, $p<0.0001)$. There was a slight, but significant, increase in the FPF delivered via the Diamond VHC compared with the AC Z-Stat VHC $(p=0.0049)$. At $30 \mathrm{~L} / \mathrm{min}$, there were no significant between-VHC differences in the FPF of albuterol. No between-VHC differences in FPF were observed in the delivery of beclomethasone at $15 \mathrm{~L} / \mathrm{min}$ or $30 \mathrm{~L} / \mathrm{min}$.

\section{Diamond VHC; washed versus unwashed comparisons}

All comparisons of the Diamond VHC and the unwashed Diamond VHC were carried out using albuterol, at $30 \mathrm{~L} / \mathrm{min}$ flow rate. Comparison of aerosol parameters from the Diamond VHC and the unwashed Diamond VHC demonstrated no indication of differences in any of the key parameters of drug delivery (Fig. 9); for all endpoints, the $90 \%$ confidence interval values fell well within the $85 \%$ to $118 \%$ acceptance interval for in vitro equivalence. ${ }^{(11)}$

\section{Discussion}

The aim of this study was to conduct a characterization of aerosol delivery performance from the antistatic Diamond VHC and make comparisons with a second antistatic VHC

Table 7. Estimated Mean Absolute Difference in Dose to VHC (\% Label Claim), and 90\% CONFIDENCE Limits, By pMDI PRODUCt, Flow Rate, and VHC COMPARISON

\begin{tabular}{|c|c|c|c|c|c|c|}
\hline $\begin{array}{l}\text { pMDI } \\
\text { product }\end{array}$ & $\begin{array}{c}\text { Flow } \\
\text { rate } \\
\text { (L/min) }\end{array}$ & Device comparison & $\begin{array}{l}\text { Estimated } \\
\text { difference } \\
(\% \text { LC) }\end{array}$ & $\begin{array}{l}\text { Lower } 90 \% \\
\text { confidence } \\
\text { limit }(\%)\end{array}$ & $\begin{array}{l}\text { Upper } 90 \% \\
\text { confidence } \\
\text { limit }(\%)\end{array}$ & $\mathrm{p}$ value \\
\hline \multirow[t]{2}{*}{ Albuterol } & 15 & $\begin{array}{l}\text { AC Z-Stat vs. Diamond } \\
\text { AC Plus vs. Diamond } \\
\text { AC Plus vs. AC Z-Stat }\end{array}$ & $\begin{array}{r}2.9 \\
12.7 \\
9.9\end{array}$ & $\begin{array}{r}-0.1 \\
9.8 \\
6.9\end{array}$ & $\begin{array}{r}5.9 \\
15.7 \\
12.8\end{array}$ & $\begin{array}{l}0.1086 \\
0.0008 \\
0.0021\end{array}$ \\
\hline & 30 & $\begin{array}{l}\text { AC Z-Stat vs. Diamond } \\
\text { AC Plus vs. Diamond } \\
\text { AC Plus vs. AC Z-Stat }\end{array}$ & $\begin{array}{r}-4.7 \\
0.7 \\
5.4\end{array}$ & $\begin{array}{r}-6.9 \\
-1.7 \\
3.1\end{array}$ & $\begin{array}{r}-2.4 \\
3.1 \\
7.6\end{array}$ & $\begin{array}{l}0.0030 \\
0.6236 \\
0.0011\end{array}$ \\
\hline \multirow[t]{2}{*}{ Beclomethasone } & 15 & $\begin{array}{l}\text { AC Z-Stat vs. Diamond } \\
\text { AC Plus vs. Diamond } \\
\text { AC Plus vs. AC Z-Stat }\end{array}$ & $\begin{array}{r}-0.1 \\
6.4 \\
6.5\end{array}$ & $\begin{array}{r}-4.9 \\
1.6 \\
1.7\end{array}$ & $\begin{array}{r}4.7 \\
11.2 \\
11.3\end{array}$ & $\begin{array}{l}0.9718 \\
0.0370 \\
0.0348\end{array}$ \\
\hline & 30 & $\begin{array}{l}\text { AC Z-Stat vs. Diamond } \\
\text { AC Plus vs. Diamond } \\
\text { AC Plus vs. AC Z-Stat }\end{array}$ & $\begin{array}{r}0.8 \\
-0.8 \\
-1.6\end{array}$ & $\begin{array}{l}-2.0 \\
-3.6 \\
-4.5\end{array}$ & $\begin{array}{l}3.7 \\
2.1 \\
1.2\end{array}$ & $\begin{array}{l}0.6056 \\
0.6239 \\
0.3244\end{array}$ \\
\hline
\end{tabular}

$\%$ LC, \% label claim. 


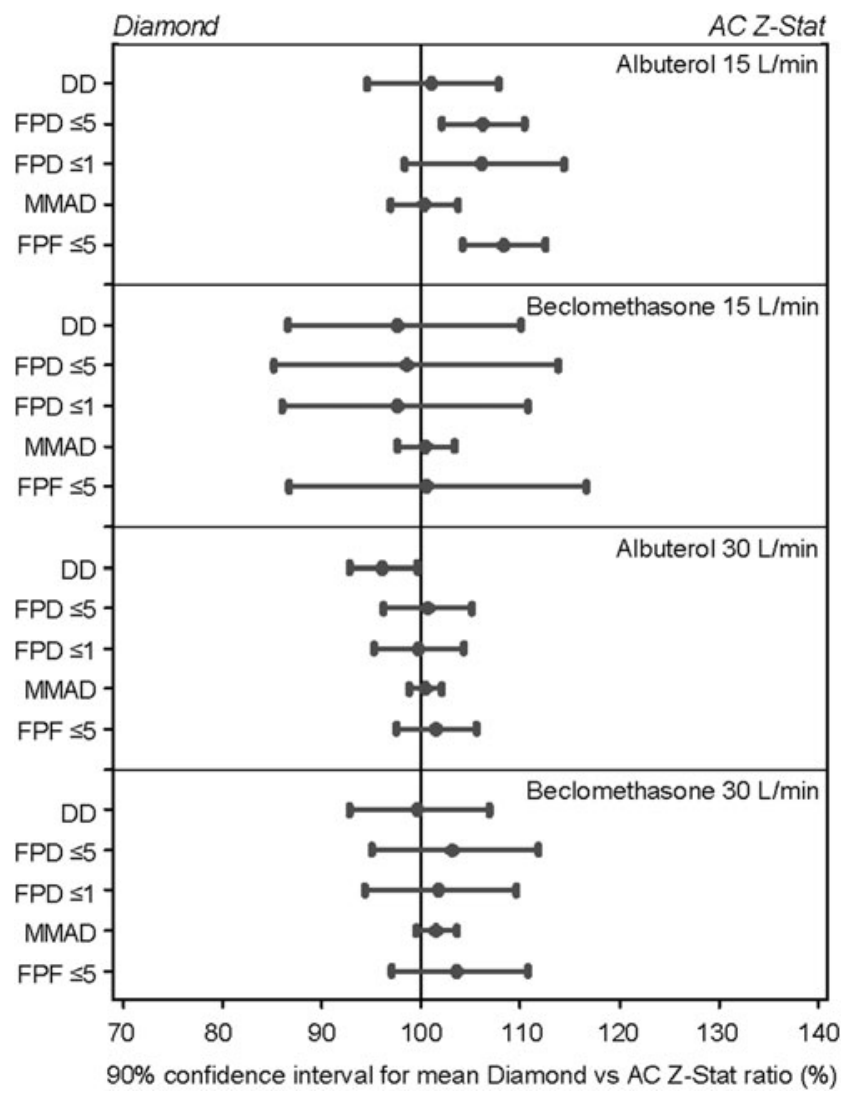

FIG. 6. Forest plots of $90 \%$ confidence intervals for the relative difference by pMDI drug formulation and key aerosol parameters; Diamond VHC versus AC Z-Stat VHC. $\mathrm{DD}$, delivered dose.

(AC Z-Stat VHC) and a conventional VHC (AC Plus VHC). Using in vitro test methods, key delivery parameters that are typically used to define the characteristics of aerosols and aerosol delivery devices were assessed. The experiments were carried out at different flow rates using two different pMDI drug formulations that are commonly prescribed to respiratory patients.

\section{pMDI alone versus VHC comparison}

The data presented here reflect the expected pattern of a greater delivered dose when the pMDIs were used alone, and a lower delivered dose when the pMDIs were used with a VHC. Of the pMDI-VHC pairs tested here, an equivalent delivered dose of QVAR was achieved via the two antistatic VHCs, compared with the pMDI alone, when the lower flow rate of $15 \mathrm{~L} / \mathrm{min}$ was used. In contrast, the conventional AC Plus VHC delivered a significantly lower dose (electrostatic effects may have contributed to this difference). This indicates that exceptions to the expected pattern of drug delivery may occur for certain pMDI formulations, under certain flow rates.

The presence of a VHC, compared with the pMDI alone, made little difference to the MMAD. Given the significant differences observed for FPD, FPD1, and FPF, which might be expected to be associated with changes in MMAD, this is perhaps surprising. The similar MMAD, regardless of differences in the FPD and FPF, reflects the reduction in the

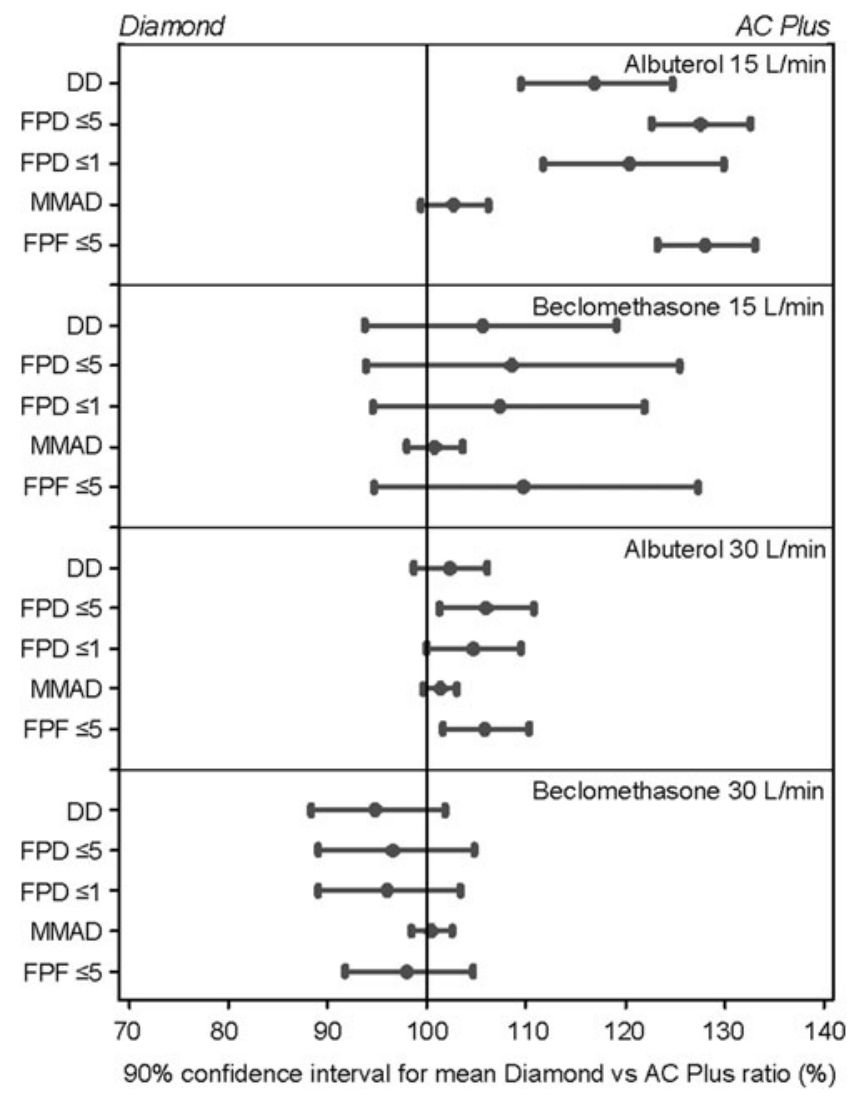

FIG. 7. Forest plots of $90 \%$ confidence intervals for the relative difference by pMDI drug formulation and key aerosol parameters; Diamond VHC versus AC Plus VHC. $\mathrm{DD}$, delivered dose.

number of large particles entering the impactor associated with use of a VHC, and the greater throat deposition associated with use of the pMDI alone. The result is that, despite higher deposition in the presence of a VHC, the relative distribution pattern is very similar, and subsequently the MMAD is comparable.

The difference observed was in terms of the quality of aerosol; when a VHC was used, there was a tendency toward the delivery of a greater FPD, and greater FPF, particularly at the $15 \mathrm{~L} / \mathrm{min}$ flow rate, compared with the pMDIs alone (Figs. 2 and 4). As the use of a VHC was associated with either a reduction in delivered dose or no change in delivered dose, it can be assumed that the amount of drug contained in coarse particles was reduced. Such an effect on aerosol quality is a well-recognized consequence, and one of the main advantages, of using a VHC; the evaporation of propellant from large, nonrespirable aerosol particles, during transport through the VHC, produces smaller particles with a more favorable respirable profile. In addition to potentially improving lung dose, use of a VHC has previously been shown to reduce oropharyngeal impaction of aerosol in an in vitro model, compared with a pMDI alone. ${ }^{(5)}$ This effect was most pronounced at lower $(<60 \mathrm{~L} / \mathrm{min})$ flow rates, similar to those achieved by patients inhaling tidally from a VHC.

In the present study, each pMDI showed a distinct pattern of deposition, which was maintained across the different VHCs tested. This suggests that, in addition to the delivery 


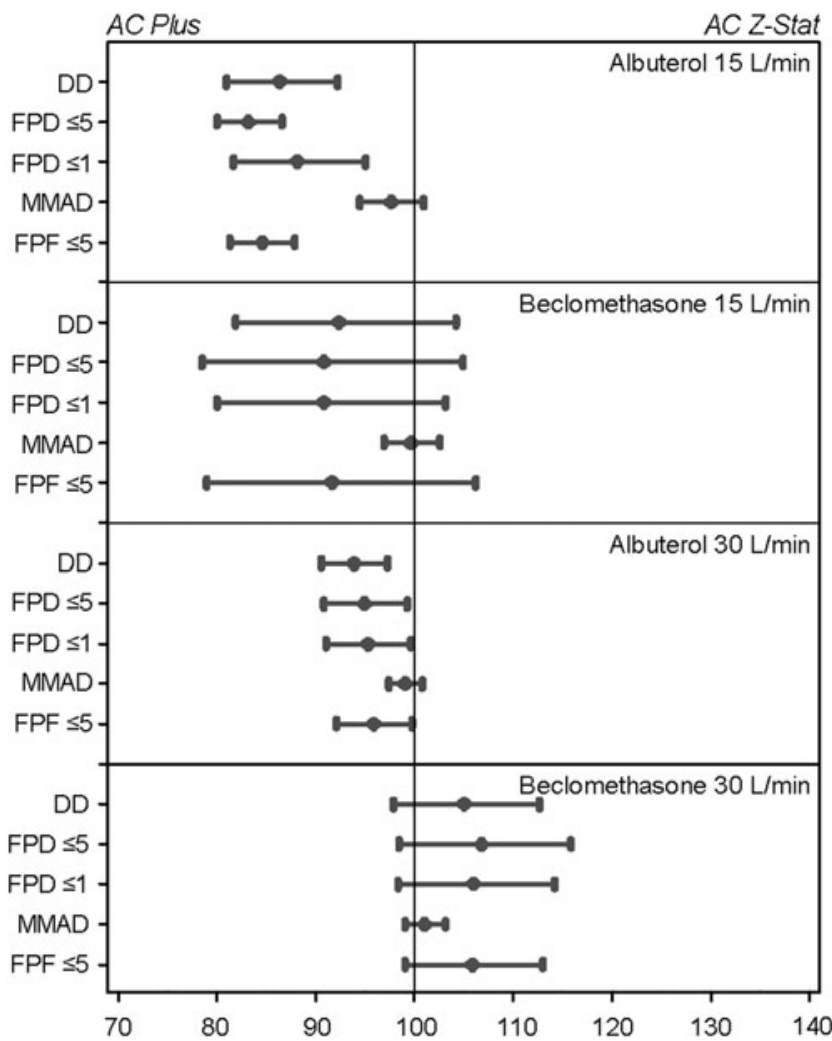

$90 \%$ confidence interval for mean AC Plus vs AC Z-Stat ratio (\%)

FIG. 8. Forest plots of $90 \%$ confidence intervals for the relative difference by pMDI drug formulation and key aerosol parameters; AC Plus VHC versus AC Z-Stat VHC. $\mathrm{DD}$, delivered dose.

method, drug formulation is an important influence on aerosol delivery.

\section{Comparison of $\mathrm{VHC}$ brands}

In terms of the key aerosol characteristics, performance of the three VHC brands was broadly comparable for the two flow rates and two different pMDI drug formulations studied. Delivery via the two antistatic VHCs (Diamond VHC and $\mathrm{AC} \mathrm{Z}$-Stat VHC) was equivalent, with the Diamond VHC delivering a slightly greater FPD and FPF of albuterol at the lower flow rate.

The characteristics of aerosol delivered via the AC Plus VHC differed from those of the aerosol delivered via the antistatic VHCs, with the exception of MMAD, which showed no significant differences between VHC brands. Similar to the effect described for the comparison of pMDI alone with VHC, this is likely to have been influenced by deposition within the NGI throat.

\section{Flow rates}

Across the two flow rates, the difference in delivered dose for each pMDI-VHC combination was generally not substantial. At a lower flow rate, the FPD, FPD1, and FPF appeared to be affected to a greater degree compared with the other parameters assessed. This was particularly the case when the drug was delivered via the pMDI alone, or using the conventional VHC, the AC Plus VHC. This is consistent with recent work from our group, which suggested that, when a conventional "static" VHC is used, drug delivery may be more vulnerable to the effect of inhalation delays, particularly at lower flow rates. ${ }^{(12)}$ Notably, previous work comparing the lung deposition achieved by inhaling via a pMDI with VHC using a breathing maneuver and tidal breathing found that some benefit was conferred by undertaking the breathing maneuver. ${ }^{(3)}$ However, the previous study used the conventional AC Plus VHC. It is plausible that the lower flow rates likely to be associated with tidal breathing, compared with a maximal inhalation maneuver, may have less impact on lung dose if an antistatic VHC were used.

The lower flow rate had a comparable influence on output from the two antistatic VHCs. Differences in parameters were less pronounced with the antistatic VHCs than those observed with the pMDI alone or the AC Plus VHC, with the FPD1 being the most affected. Low flow rate generally appeared to influence the delivery of beclomethasone to a greater degree than the delivery of albuterol. This suggests that the solution formulation, which had a much lower MMAD than the suspension formulation, may be particularly sensitive to low inhalation flow. QVAR, the solution of beclomethasone used in the present study, is a unique formulation, with a low MMAD. Previous literature has indicated that, unlike a larger particle chlorofluorocarbon (CFC) beclomethasone formulation, the delivery of QVAR may not be affected by the use of a VHC, with or without an inhalation delay. ${ }^{(13)}$ It is not known whether the use of a steroid formulated as a suspension, with a corresponding larger MMAD, would influence the results of the present study.

\section{Washed versus unwashed Diamond VHC}

The results of this study indicated that the performance of the Diamond VHC, when taken out of its original packaging
FIG. 9. Forest plots of $90 \%$ confidence intervals for relative differences between the Diamond VHC versus unwashed Diamond VHC for key aerosol parameters. DD, delivered dose.

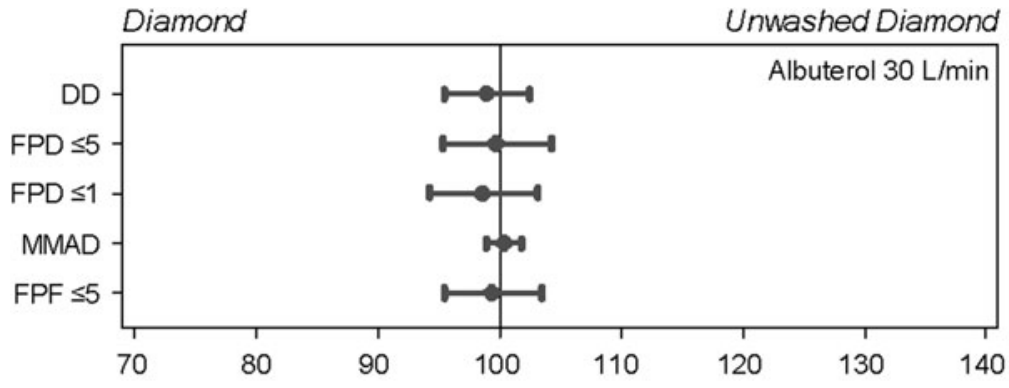

$90 \%$ confidence interval for mean Diamond vs unwashed Diamond ratio (\%) 
and used for the first time, was not influenced by whether it was in a washed or unwashed state. A newly opened Diamond VHC can therefore be used immediately, without any washing required before initial use.

\section{Limitations of the study}

The study test plan was not fully randomized due to a lack of Diamond VHC prototypes, meaning that the tests on the washed Diamond VHC had to be conducted after the unwashed tests. However, as unwashed tests were not conducted on the other VHC brands, this prevented the use of a balanced test matrix.

The interpretation of the two inhalation flow rates deserves further consideration; the "high" flow rate of $30 \mathrm{~L} /$ min, which achieved a more positive respirable profile than the lower flow rate, has, in a previous lung deposition study, been classed as a "low" inhalation flow. ${ }^{(14)}$ This would suggest that, in order to adequately approximate likely drug delivery to patients, in whom excessively rapid inhalation flow rates are well-documented, it would be necessary to use a wider range of higher flow rates. It should be noted, however, that the previous study was conducted using old CFC-containing pMDIs; it is likely that contemporary HFA formulations exhibit different properties. Indeed, recent in vitro work suggests that the benefit of VHC use in reducing oropharyngeal deposition becomes less pronounced at higher flow rates, and is canceled out completely once a flow rate of $60 \mathrm{~L} / \mathrm{min}$ is reached. ${ }^{(5)}$

Given that patients are known to struggle to comply with correct pMDI and VHC technique in real life, the generalizability of the current results is likely to be limited. It is well recognized, for example, that patients using a pMDI with a VHC will often unwittingly introduce an inhalation delay between actuation of the pMDI and inhalation from the VHC mouthpiece. Such inhalation delays can reduce the respirable drug available for inhalation. Although not explored in the current article, we have recently undertaken an extensive in vitro analysis of the effects of inhalation delays. ${ }^{(12)}$ This analysis, which used a test setup similar to that of the present article, indicated that emitted dose increased with flow rate and decreased with lengthier inhalation delays. The antistatic VHCs tested were shown to have in vitro equivalence, whereas the conventional $\mathrm{VHC}$ had a more pronounced flow rate dependence. ${ }^{(12)}$

Separate from any effect of poor compliance, environmental factors, including extremes of temperature and humidity, are known to affect the performance of solution and suspension HFA pMDIs. ${ }^{(15)}$ The current study was undertaken in ambient laboratory conditions, and therefore results may not be generalizable to other environments.

Finally, it is widely recommended that patients, particularly the very young and those administering high doses of inhaled corticosteroids, use a VHC with facemask. Results of the present study cannot be extrapolated to tests in which a VHC is used with a facemask, rather than the VHC with mouthpiece.

\section{Conclusions}

Overall, compared with the pMDI alone, the use of a pMDI with VHC influenced the dose of aerosol within the respirable range, particularly at low flow rates. Between-
VHC comparisons showed that the two antistatic VHCs, the Diamond $\mathrm{VHC}$ and the AC Z-Stat VHC, achieved in vitro equivalence. Between the VHCs tested, the FPD, FPD1, and FPF were greater for albuterol when the drug was delivered via the antistatic Diamond and AC Z-Stat VHCs compared with the conventional AC Plus VHC. These results indicate that use of an antistatic VHC facilitates the output of aerosol with a better aerodynamic particle size distribution compared with that available from a conventional VHC. This effect is more apparent at lower flow rates and appears to be dependent on the pMDI drug or formulation. Similarly, when the within-VHC effect of different flow rates was compared for albuterol and beclomethasone, the effect appeared to be dependent on both the VHC and the pMDI drug or formulation.

Results also indicated distinct patterns of drug delivery for the two pMDIs tested. Although there were no betweenVHC differences in the delivery of beclomethasone, in any of the key aerosol parameters and at either of the two flow rates, the properties of albuterol aerosol were influenced by the material from which the VHC was made.

\section{Acknowledgments}

The authors acknowledge Adam Metcalf of Respironics Respiratory Drug Delivery (UK) Ltd., a business of Philips Electronics UK Limited, Chichester, West Sussex, UK, for his analytical assistance, and Kurt Nikander for his technical assistance and guidance. We also acknowledge PS5 Consultants Ltd., Portsmouth, UK, for drafting (Clare Nicholls) and editorial assistance (Stephen Keen). The study was sponsored by Respironics Respiratory Drug Delivery (UK) Ltd.

\section{Author Disclosure Statement}

Ross Hatley, Dirk von Hollen, and Lois Slator are employees of Respironics Respiratory Drug Delivery (UK) Ltd. Dennis Sandell is a self-employed statistician and the owner of S5 Consulting. Dennis is an independent consultant who was engaged by Philips on this project.

\section{References}

1. Nikander K, Nicholls C, Denyer J, and Pritchard J: The evolution of spacers and valved holding chambers. J Aerosol Med Pulm Drug Deliv. 2014;27:S-4-S-23.

2. Foss SA, and Keppel JW: In vitro testing of MDI spacers: a technique for measuring respirable dose output with actuation in-phase or out-of-phase with inhalation. Respir Care. 1999;44:1474-1485.

3. Roller CM, Zhang G, Troedson RG, Leach CL, Le Souëf PN, and Devadason SG: Spacer inhalation technique and deposition of extrafine aerosol in asthmatic children. Eur Respir J. 2007;29:299-306.

4. Devadason SG, Huang T, Walker S, Troedson R, and Le Souëf PN: Distribution of technetium-99m-labelled QVAR delivered using an Autohaler device in children. Eur Respir J. 2003;21:1007-1011.

5. Rahmatalla MF, Zuberbuhler P, Lange $\mathrm{C}$, and Finlay W: In vitro effect of a holding chamber on the mouth-throat deposition of $\mathrm{QVAR}^{\circledR}$ (hydrofluoroalkane-beclomethasone dipropionate). J Aerosol Med. 2002;15:379-385.

6. Newman SP, Millar AB, Lennard-Jones TR, Morén F, and Clarke SW: Improvement of pressurised aerosol de- 
position with Nebuhaler spacer device. Thorax. 1984;39: 935-941.

7. Holzner PM, and Muller BW: An in vitro evaluation of various spacer devices for metered-dose inhalers using the Twin Impinger. Int J Pharm. 1994;106:69-75.

8. Chew NY, and Chan HK: The effect of spacers on the delivery of metered dose aerosols of nedocromil sodium and disodium cromoglycate. Int J Pharm. 2000;200:87-92.

9. Mitchell JP, Coppolo DC, and Nagel MW: Electrostatics and inhaled medications: influence on delivery via pressurized metered-dose inhalers and add-on devices. Respir Care. 2007;52:283-300.

10. British Standards Institution: BS EN 13544-1:2007+ A1:200: Respiratory therapy equipment. Nebulizing systems and their components. British Standards Institution, London; 2007.

11. European Medicines Agency/Committee for Medicinal Products for Human Use (CHMP): Guideline on the requirements for clinical documentation for orally inhaled products (OIP) including the requirements for demonstration of therapeutic equivalence between two inhaled products for use in the treatment of asthma and chronic obstructive pulmonary disease (COPD) in adults and for use in the treatment of asthma in children and adolescents. European Medicines Agency, London, 22 January 2009. Doc. Ref. CPMP/EWP/4151/00 Rev. 1.

12. Slator L, von Hollen D, Sandell D, and Hatley RHM: In vitro comparison of the effect of inhalation delay and flow rate on the emitted dose from three valved holding chambers. J Aerosol Med Pulm Drug Deliv. 2014;27:S-37-S-43.

13. Leach CL, and Collis GL: A pilot study to assess lung deposition of HFA beclomethasone and CFC-beclomethasone from a pressurized metered dose inhaler with and without add-on spacers, and using varying breathhold times. J Aerosol Med Pulm Drug Deliv. 2010;23:355-361.

14. Newman SP, Pavia D, Garland N, and Clarke SW: Effects of various inhalation modes on the deposition of radioactive pressurized aerosols. Eur J Respir Dis. 1982;63(Suppl 119):57-65.

15. Shemirani FM, Hoe S, Lewis D, Church T, Vehrigh R, and Finlay W: In vitro investigation of the effect of ambient humidity on regional delivered dose with solution and suspension MDIs. J Aerosol Med Pulm Drug Deliv. $2013 ; 26: 215-222$.

Received on June 6, 2013 in final form, December 6, 2013

Reviewed by:

Chet Leach

Jan Olof Svensson

Address correspondence to: Dr. Ross H.M. Hatley Head of Aerosol Science Respironics Respiratory Drug Delivery (UK) Ltd., a business of Philips Electronics UK Limited

Chichester Business Park City Fields Way

Tangmere Chichester

West Sussex PO20 2FT, UK

E-mail: ross.hatley@philips.com 